\title{
Influence of Initial Crystallization Temperature of Form II on the Nucleation and Growth of Form I IPBu Crystals during II-I Phase Transition
}

Rui Xin, ${ }^{1}$ Yunpeng Li, ${ }^{1}$ Zhixin Guo, ${ }^{2}$ Jian Hu, ${ }^{1}$ Shaojuan Wang, ${ }^{1}$ Hao Zhang, ${ }^{1}$

Meiling Xue, ${ }^{1 *}$ Xiaoli Sun $^{2 *}$ and Shouke Yan, ${ }^{1,2 *}$

${ }^{1}$ Key Laboratory of Rubber-Plastics, Qingdao University of Science \& Technology, Qingdao 266042, China

${ }^{2}$ State Key Laboratory of Chemical Resource Engineering, Beijing University of Chemical Technology, Beijing, 100029, China

E-mails: meilingxue@qust.edu.cn; xiaolisun@mail.buct.edu.cn; and skyan@qust.edu.cn or skyan@mail.buct.edu.cn

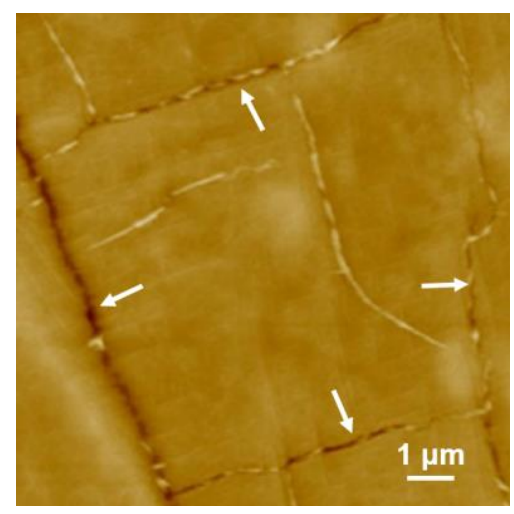

Figure S1. An enlarged part of the AFM height image shown in Figure $2 b$ of the $\mathrm{iPBu}$ thin film crystallized isothermally at $100{ }^{\circ} \mathrm{C}$. The arrows show the gaps caused by depletion. 

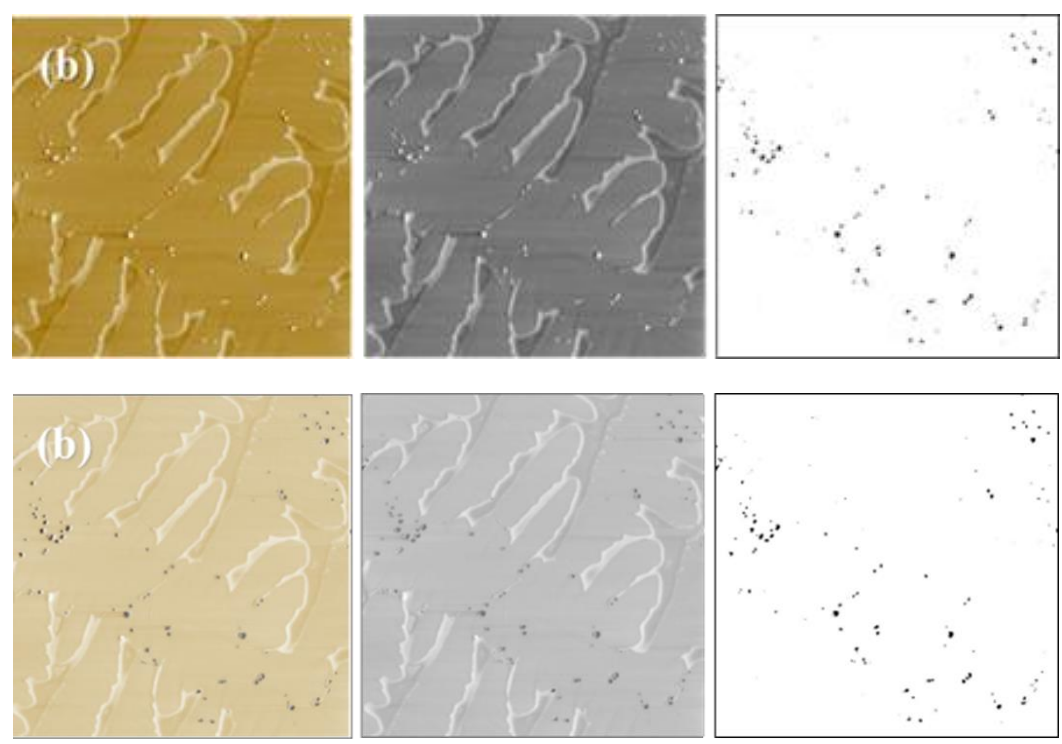

Figure S2. Superimposing the obtained form I crystal distribution image (top right) with AFM phase (top left) and 8-bit grayscale (top middle) images shows the good overlap of form I crystals (bottom panel) and results in the convert of contrast. This demonstrates the feasibility of our used image treatment technique for analyzing the II-I phase transition of iPBu.

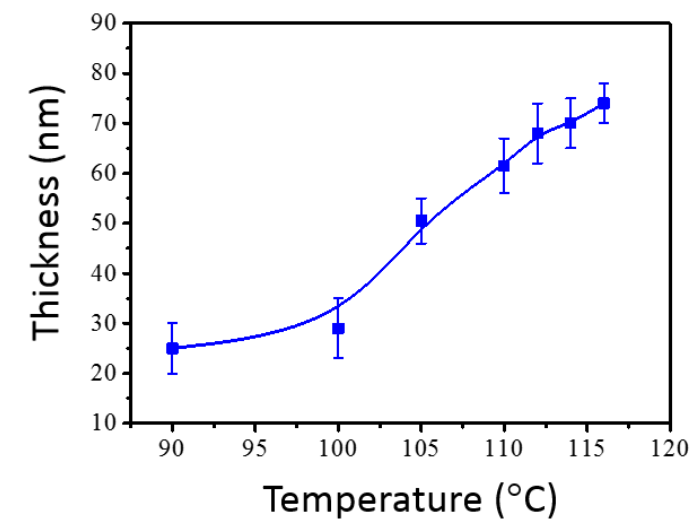

Figure S3. Plot of thickness of iPBu form II crystals, defined as the distance between the silicon wafer and crystal top surface measured by AFM height images, against the crystallization temperature. 